\title{
FLOW SEPARATION PHENOMENA FOR STEADY FLOW OVER A CIRCULAR CYLINDER AT LOW REYNOLDS NUMBER
}

\author{
Toukir Islam ${ }^{1}$, S.M. Rakibul Hassan ${ }^{2}$ and Mohammad Ali ${ }^{3}$ \\ Department of Mechanical Engineering, BUET, Dhaka, Bangladesh. \\ ${ }^{1}$ Email: toukir.buet07@gmail.com
}

\begin{abstract}
Flow separation is one of the major distinguishing phenomena between the theoretical or potential flow and actual fluid flow that introduces several aerodynamic forces and other renowned phenomena. Here in this article, different flow separation phenomena such as onset of separation, angular position of the separation point, size of the separation bubble, drag due to the separation and effect of the blockage ratio on the position of the separation point for steady flow over a smooth circular cylinder are studied numerically using the finite volume method at very low Reynolds number up to 50 , and the trends of change of these phenomena with increasing Reynolds number are expressed in the form of some empirical equations and compared with results of previously published literature. The separation starts at Reynolds numbers as low as 2.0 and the starting-separation angle is $146.557^{\circ}$ from the upstream stagnation point, while with increasing Reynolds number, the point of separation travels upstream and the separation bubble length increases. The profound effect of the blockage ratio on the location of the separation point is evident in this numerical analysis. Favre-averaged Navier-Stokes equations, a k- $\varepsilon$ turbulence model and the finite volume method are used for numerical analysis, in which heat transfer or generation, and buoyancy effects are not taken in account.
\end{abstract}

Keywords: flow separation; separation angle; separation bubble; empirical equations.

\section{INTRODUCTION}

Flow over circular cylinders has been one of the canonical research topics over the years for fluid dynamists and engineers, not only because of its geometrical simplicity but also for its practical importance. The problem of viscous steady flow past a circular cylinder has for a long time received considerable attention among fluid dynamists such as Fornberg (1980), Sen, Mittal, and Biswas (2010), Wu et al. (2004), Park, Kwon, and Choi (1998), Taneda (1956) etc. Different researchers have shaped the existing knowledge regarding the steady flow at low Reynolds number $(R e)$. Fornberg (1980) studied the steady viscous flow past a circular cylinder for Reynolds numbers up to 300 . Sen et al. (2010) studied the steady flow at the range of $\operatorname{Re} \leq 40$ using the finite element method and described the effect of blockage ratio, position of separation on surface, separation bubble size etc. and expressed these phenomena in terms of empirical equations which are a function of $R e$. Wu et al. (2004) introduced soap-film flow visualization instead of the commonly used streakline image from finite time exposure to obtain the time averaged separation angle. They also described different separation phenomena as functions of $R e$. Park et al. (1998) used high resolution calculation for the flow over a cylinder up to $R e 160$ and provided detailed information on the flow quantities on the cylinder surface at low Re. But according to many researchers like Sen 
et al. (2010), the flow over a circular cylinder becomes unsteady around Reynolds number 50. The first wake instability, manifestation of a Hopf bifurcation, occurs at $R e$ around 47, according to Williamson (1996). So, steady flow approximation after $R e 50$ would lead to inaccurate results.

The Reynolds number can be considered as the measure-stick for different flow phenomena changes in the study of flow over a cylinder. At low Reynolds number, the flow can be considered steady, while with increasing $R e$ the symmetry between the upstream and downstream disappears as the flow becomes unsteady. Flow separation occurs at very low $R e$ - well below 10 . Flow separation is characterized by detachment of streamlines from the cylinder surface usually at the downstream of the cylinder (Fornberg, 1980). This occurs when the boundary layer travels far enough against an adverse pressure gradient that the speed of the boundary layer relative to the object falls to almost zero. Physically a separated flow develops when the fluid element comes to a stop somewhere downstream and reverses its direction. The point from which the flow is separated from the surface of the cylinder is the separation point and will be denoted as $S$ for the rest of the article. The angular position of $S$, i.e. separation angle $\left(\theta_{S}\right)$, changes with $R e$. The flow separated from the shoulder of the cylinder, both on the upper and lower surface, is reattached at the downstream of the cylinder. Thus a bubble of separated flow is created due to symmetric eddies just behind the cylinder, and this is called the separation bubble. The separation bubble's length and width bears significant information about the flow characteristics, aerodynamic forces on the cylinder, intensity and position of separation, etc. Sen et al. (2010) described these phenomena with respect to different blockage ratios (BR), which is actually the ratio of the cylinder diameter to the height of the computational domain, whereas some authors like Wu et al. (2004) suggested that the blockage ratio is not a major reason for discrepancy among various experiments at higher $R e$, but is significant for variation in the separation angle at low $R e$. The present study is concentrated on the separation phenomena and phenomena that are directly affected by the modes and positions of separation. The angular position of separation $\left(\theta_{S}\right)$, variation of the separation angle with $R e$ and blockage ratio $(B R)$, separation bubble length and symmetric eddies enclosed by the bubble, drag on the cylinder, etc. are emphasised. These phenomena are expressed in the form of empirical equations and as functions of $R e$. As steady flow is limited by $R e$ around 50, our study is limited to this range.

\section{NUMERICAL PROCEDURE}

\section{Governing Equations}

Favre-averaged Navier-Stokes equations are used, where time-averaged effects of the flow turbulence on the flow parameters are considered, whereas the other, i.e. large-scale, timedependent phenomena are taken into account directly. Through this procedure, extra terms known as the Reynolds stresses appear in the equations, for which additional information must be provided. To close this system of equations, the flow simulation procedure employs transport equations for the turbulent kinetic energy and its dissipation rate, the socalled k- $\varepsilon$ model. One system of equations is employed to describe both laminar and turbulent flows, and transition from a laminar to turbulent state or vice versa is possible. The conservation laws for mass, angular momentum and energy in the Cartesian coordinate system rotating with angular velocity $\Omega$ about an axis passing through the coordinate system's origin can be written in the conservation form as follows: 


$$
\begin{gathered}
\frac{\partial \rho}{\partial t}+\frac{\partial\left(\rho u_{i}\right)}{\partial x_{i}}=0 \\
\frac{\partial\left(\rho u_{i}\right)}{\partial t}+\frac{\partial\left(\rho u_{i} u_{j}\right)}{\partial x_{j}}+\frac{\partial \mathrm{P}}{\partial x_{i}}=\frac{\partial}{\partial x_{i}}\left(\tau_{i j}+\tau_{i j}{ }^{R}\right)+S_{i} \\
\frac{\partial(\rho H)}{\partial t}+\frac{\partial\left(\rho u_{i} \mathrm{H}\right)}{\partial x_{i}}=\frac{\partial}{\partial x_{i}}\left[u_{j}\left(\tau_{i j}+\tau_{i j}{ }^{R}\right)+q_{i}\right]+\frac{\partial \rho}{\partial t}+S_{i} u_{i}-\tau_{i j}{ }^{R} \frac{\partial u_{i}}{\partial x_{i}}+\rho \varepsilon+Q_{H} \\
H=h+\frac{u^{2}}{2}
\end{gathered}
$$

Here $u$ is fluid velocity, $\rho$ is fluid density, $S_{i}$ is a mass-distributed external force per unit mass due to porous media resistance, a buoyancy $\left(-\rho g_{i}\right)$, and the coordinate system's rotation, $h$ is the thermal enthalpy, $Q_{H}$ is a heat source or sink per unit volume, $\tau_{i j}$ is the viscous shear stress tensor, $q_{i}$ is the diffusive heat flux. The subscripts are used to denote summation over the three coordinate directions.

Now for Newtonian fluids, the viscous shear stress tensor is defined as

$$
\tau_{i j}=\mu\left(\frac{\partial u_{i}}{\partial x_{j}}+\frac{\partial u_{j}}{\partial x_{i}}-\frac{2}{3} \delta_{i j} \frac{\partial u_{k}}{\partial x_{k}}\right)
$$

According to the Boussinesq assumption, the Reynolds-stress tensor has the following form:

$$
\tau_{i j}^{R}=\mu_{t}\left(\frac{\partial u_{i}}{\partial x_{j}}+\frac{\partial u_{j}}{\partial x_{i}}-\frac{2}{3} \delta_{i j} \frac{\partial u_{k}}{\partial x_{k}}\right)-\frac{2}{3} \rho k \delta_{i j}
$$

Here $\delta_{i j}$ is the Kronecker delta function, which is equal to unity when $\mathrm{i}=\mathrm{j}$ and zero otherwise. $\mu$ is the dynamic viscosity coefficient, $\mu_{t}$ is the turbulent eddy viscosity coefficient and $k$ is the turbulent kinetic energy. A point to be noted is that both $k$ and $\mu_{t}$ are zero for laminar flow. In the frame of the $k-\varepsilon$ turbulence model, $\mu_{t}=f_{\mu} \frac{C_{\mu} \rho k^{2}}{\varepsilon}$; $f_{\mu}$ here is the turbulent viscosity factor, defined as, $f_{\mu}=\left[1-\exp \left(-0.025 R_{y}\right)\right]^{2} \times[1+$ $\left.\frac{20.5}{R_{T}}\right]$; where $R_{T}=\frac{\rho k^{2}}{\mu \varepsilon}, R_{y}=\frac{\rho k^{0.5}}{\mu} \times y$

Here $y$ is the distance from the wall. This function of $f_{\mu}$ allows us to take into account laminar-turbulent transition. Two additional transport equations are used to describe the turbulent kinetic energy and dissipation at steady state:

$$
\begin{gathered}
\frac{\partial}{\partial x_{j}}\left(\rho u_{i} k\right)=\frac{\partial}{\partial x_{i}}\left[\left(\mu+\frac{\mu_{t}}{\sigma_{k}}\right) \frac{\partial k}{\partial x_{i}}\right]+\tau_{i j}{ }^{R} \times \frac{\partial u_{i}}{\partial x_{j}}-\rho \varepsilon+\mu_{t} P_{B} \\
\frac{\partial}{\partial x_{j}}\left(\rho u_{i} \varepsilon\right)=\frac{\partial}{\partial x_{i}}\left[\left(\mu+\frac{\mu_{t}}{\sigma_{\varepsilon}}\right) \frac{\partial k}{\partial x_{i}}\right]+C_{\varepsilon 1} \frac{\varepsilon}{k}\left[f_{1} \times \tau_{i j}{ }^{R} \times \frac{\partial u_{i}}{\partial x_{j}}+\mu_{t} C_{B} P_{B}\right]-C_{\varepsilon 2} f_{2} \frac{\rho \varepsilon^{2}}{k}
\end{gathered}
$$

Here $f_{1}=1+\left(\frac{0.05}{f_{\mu}}\right)^{3}, f_{2}=1-\exp \left(-R_{T}{ }^{2}\right), P_{B}=-\frac{g_{i}}{\sigma_{B}} \times \frac{1}{\rho} \frac{\partial \mathrm{P}}{\partial x_{i}}$

Now for these equations $C_{B}$ is unity when $P_{B}>0$ and zero otherwise. $\sigma_{B}=0.9, C_{\mu}=$ $0.09, C_{\varepsilon 1}=1.44, C_{\varepsilon 2}=1.92, \sigma_{k}=1, \sigma_{\varepsilon}=1.3$. These values are found empirically. These equations describe both laminar and turbulent flow. 


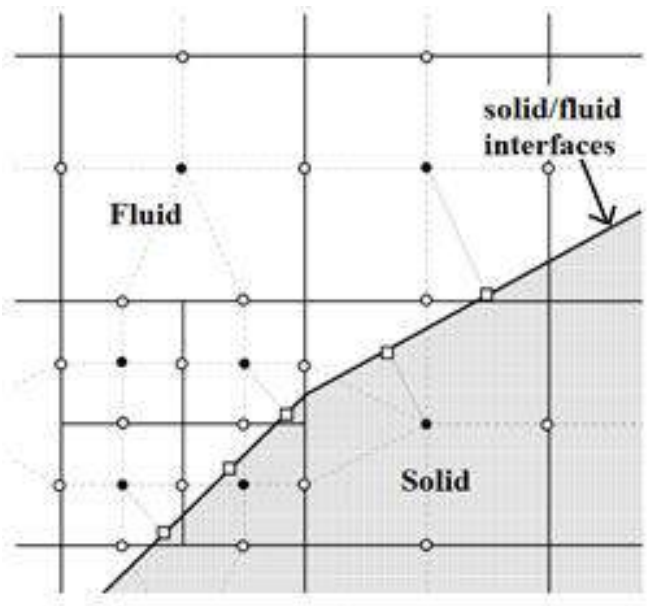

Figure 1. Computational mesh.

\section{Computational Mesh}

The rectangular computational domain is constructed such that it encloses the solid body and has the boundary planes orthogonal to the specified axes of the Cartesian coordinate system (Figure 1). Then, the computational mesh is constructed in the following several stages. First of all, a basic mesh is constructed. For that, the computational domain is divided into slices by the basic mesh planes, which are evidently orthogonal to the axes of the Cartesian coordinate system. The basic mesh is determined solely by the computational domain and does not depend on the solid/fluid interfaces. Then, the basic mesh cells intersecting with the solid/fluid interface are split uniformly into smaller cells in order to capture the solid/fluid interface with mesh cells of the specified size (with respect to the basic mesh cells). The following procedure is employed: each of the basic mesh cells intersecting with the solid/fluid interface is split uniformly into 8 child cells; each of the child cells intersecting with the interface is in turn split into 8 cells of the next level, and so on, until the specified cell size is attained. At the next stage of meshing, the mesh obtained at the solid/fluid interface with the previous procedure is refined (i.e. the cells are split further or probably merged) in accordance with the solid/fluid interface curvature. The criterion to be satisfied is established as follows: the maximum angle between the normals to the surface inside one cell should not exceed a certain threshold; otherwise, the cell is split into 8 cells. Finally, the narrow channel refinements are done if necessary. As a result of all these meshing procedures, a locally refined rectangular computational mesh is obtained and then used for solving the governing equations on it.

\section{Spatial Approximations}

The cell-centered finite volume (FV) method is used to obtain conservative approximations of the governing equations on the locally refined rectangular mesh. The governing equations are integrated over a control volume which is a grid cell, and then approximated with the cell-centered values of the basic variables. The integral conservation laws may be represented in the form of the cell volume and surface integral equation:

$$
\frac{\partial}{\partial t} \int \mathrm{Udv}+\oint F \cdot d s=\int Q d v
$$


This is replaced by the discrete form $\frac{\partial}{\partial t}(U v)+\sum_{\text {cell faces }} F . s=Q v$

The second-order upwind approximations of fluxes $F$ are based on the implicitly treated modified Leonard's QUICK approximations (Roache, 1998) and the total variation diminishing (TVD) method (Hirsch, 1988).

\section{Onset of Separation}

\section{RESULTS AND DISCUSSION}

Flow separation occurs when the streamlines no longer remain stuck to the body and cause wakes near the surface. At a definite value of blockage ratio $B R=0.2377$, the wake near the downstream of the flow is visible (Figure 2(a)) at $R e=2.0$ in our study. This indicates that for numerical calculation of 2D steady flow over the smooth cylinder at blockage ratio $B R=0.23077$, the Reynolds number at which the separation starts $\left(R e_{s}\right)$ is 2.0 and the separation angle $\theta_{S}$ is $146.557^{\circ}$. A point to be noted here is that the separation angle is measured in the clockwise direction from the upstream stagnation point. Nisi and Porter (1923) found $R e_{s}=3.2$. Experimental results of Taneda (1956) conjectured that $R e_{s}$ is 5 in the case of steady flow. Different surface roughness, blockage ratios $(B R)$ or methodologies of experiments are the probable causes of deviation of $R e_{s}$ from the findings of other researchers. Subsequent wakes are visible at Reynolds numbers 4 and 6 from Figure 2(b) and Figure 2(c) respectively, which shows that with increasing $R e$, the wake created at the separation point further develops in its form.

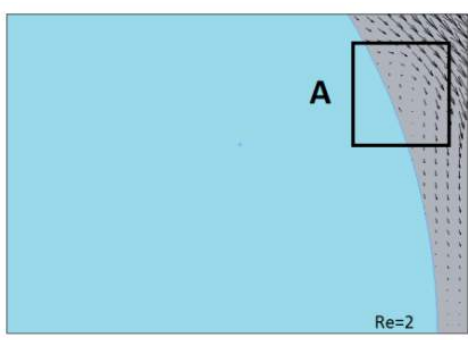

(a)

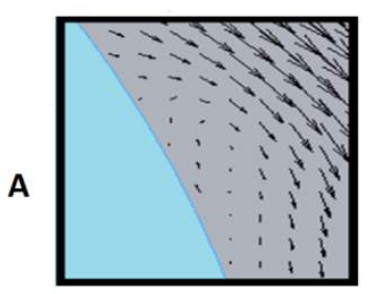

(d)

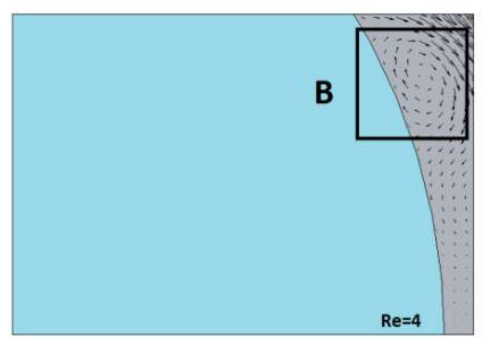

(b)

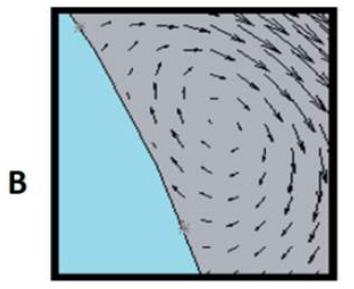

(e)

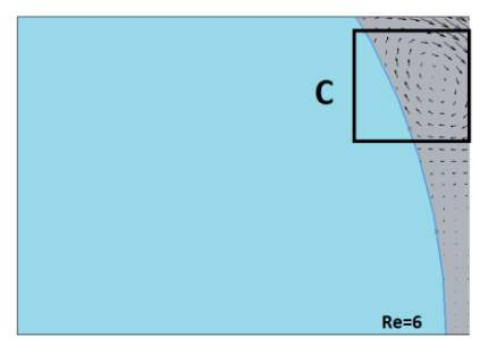

(c)

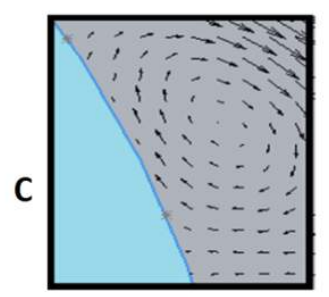

(f)

Figure 2. (a) Onset of separation at $R e=2$; development of wake at (b) $R e=4$, (c) $R e=6$; close-up view of sections (d) A, (e) B and (f) C.

\section{Effect of Blockage Ratio (BR) on Separation Angle}

The blockage ratio (BR), which is defined as the ratio of the cylinder diameter to the height of the computational domain, is significant in analyzing the separation phenomena, especially at lower Reynolds numbers. The higher the $B R$ (lower $1 / B R$ ), the lower the value of the separation angle becomes at a definite $R e$, which is evident in Figure 3. Here 
streamlines are the thicker lines and vorticity isolines are the thin lines shown on the upper-right quarter of the cylinder at different $R e$. The point $S$ denotes the separation point, and the respective separation angle and BR are presented at the lower left corner of Figure 3. Physical interpretation of the phenomena shown in Figure 3 can be presented as that the acceleration effect of the flow over the cylinder becomes more significant in magnitude when both the upper and lower walls of the computational domain approach the cylinder surface, resulting in a subsequent increase in local $R e$ which leads to earlier separation than is the case with lower $B R$ values (Sen et al., 2010). With increasing $1 / B R$ (or increasing height of the computational domain as the diameter of the cylinder is constant), the difference in the separation angle between two consecutive $1 / B R$ decreases. That means the effect of the upper and lower wall of the computational domain on the flow over the cylinder surface decreases with increasing the height of the computational domain (Sen et al., 2010).

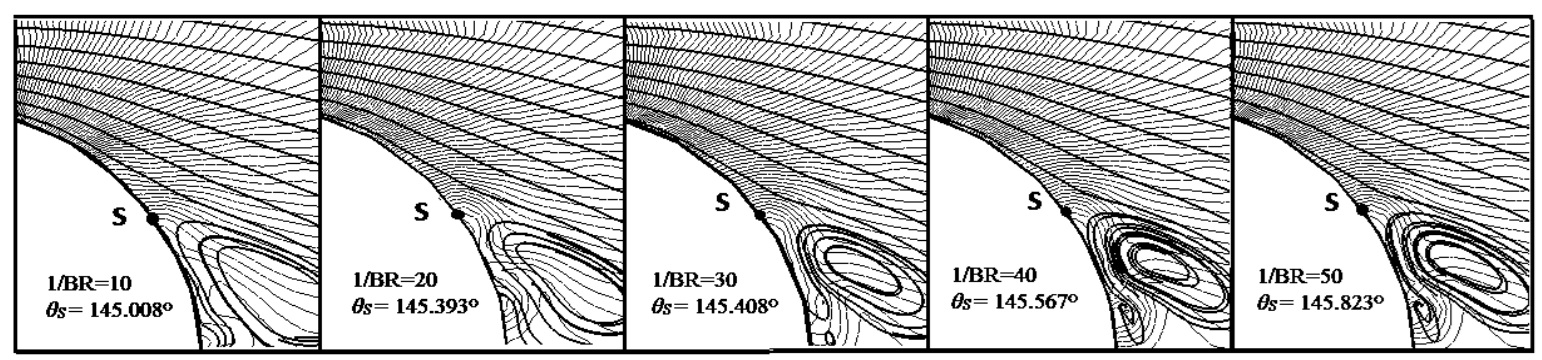

(a)

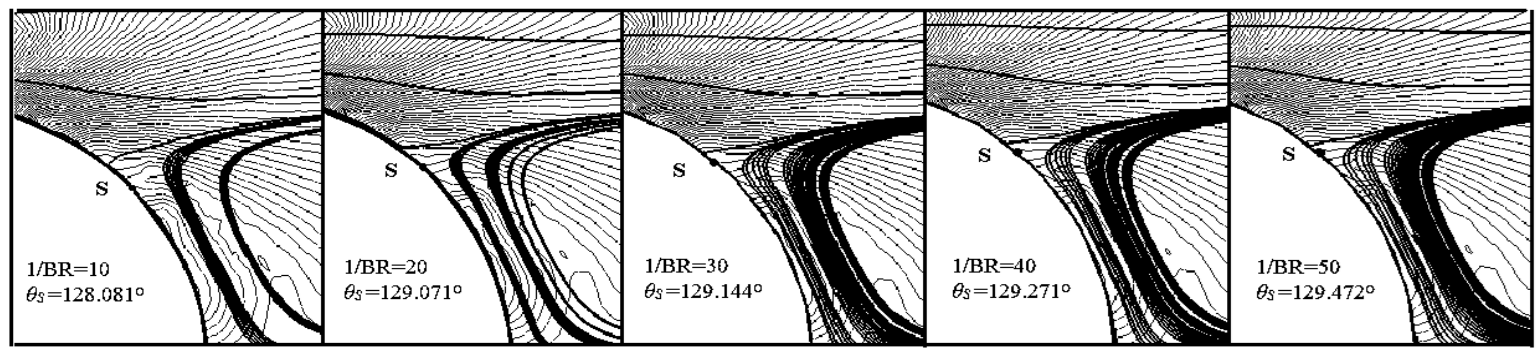

(b)

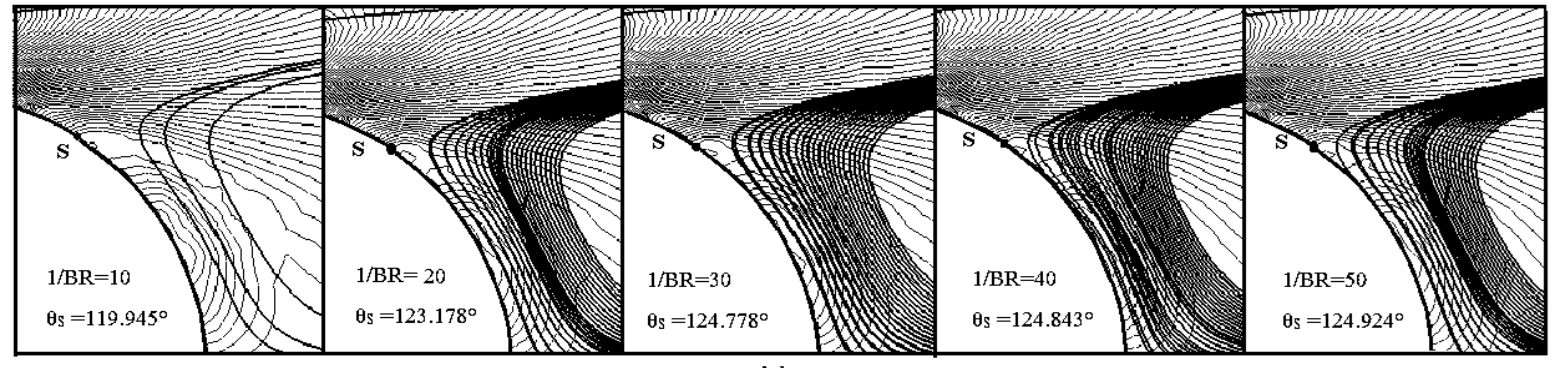

(c)

Figure 3. Effect of blockage ratio ( $B R$ ) on separation angle at (a) $R e=10$, (b) $R e=30$ and (c) $R e=50$. (Here streamlines are thicker lines and vorticity isolines are thin lines on the upperright quarter of the cylinder).

\section{Empirical Relation between Separation Angle and Reynolds Number}

The angular position of separation is a function of the Reynolds number. Figure 4 shows the relationship of $\theta_{S}$ with $R e$ and comparisons of the results with works of other researchers. It is evident that the point of separation travels upstream with increasing $R e$, 
i.e. the $\theta_{S}$ decreases with increasing $R e$. The relationship between the wake characteristics (or separation) and the Reynolds number has been frequently expressed in terms of empirical equations of either $1 / R e$ terms or $R e^{-0.5}$ terms, and the latter one is preferred by recent researchers like Wu et al. (2004), Park et al. (1998) etc. Wu et al. (2004) expressed the $\theta_{S}-R e$ relations in the first four terms for the range of $7 \leq R e \leq 200$ as

$$
\theta_{S}=95.7+267.1 R e^{-0.5}-625.9 R e^{-1}+1046.6 R e^{-3 / 2}
$$

This expression yields a root-mean-square error of 0.0004 . They also provided a simpler linear empirical equation for the range of $10 \leq R e \leq 200$ as $\theta_{S}=101.5+155.2 R e^{-0.5}$ which yields a root-mean-square error of 0.0005 . Sen et al. (2010) provided another empirical equation over the range of $10 \leq R e \leq 40$ and $0.04 \leq B R \leq 0.20$ as $\theta_{S}=77.66-152.65$ $R e^{-0.5}$ where $\theta_{S}$ is measured from the rear stagnation point. If the $\theta_{S}$ were measured from the upstream stagnation point, that equation would be $\theta_{S}=102.34+152.65 R e^{-0.5}$. From the present analysis, we found the first four terms of the relation valid for the range of $5 \leq R e \leq 50$ and $B R=0.23077$ to be

$$
\theta_{S}=85.406+317.46 R e^{-0.5}-445.85 R e^{-1}+86.264 R e^{-3 / 2}
$$

with root-mean-square error of 0.0306 and a simple empirical equation $\theta_{S}=113.21+84.165$ $R e^{-0.5}$ in the range of $10<R e \leq 50$ with root-mean-square error of 0.1658 . Though the constants of the empirical equations differ from the contributions of other researchers, the modes of the equations are the same. So the behavior of the $\theta_{S}-R e$ relation is expected to be similar.

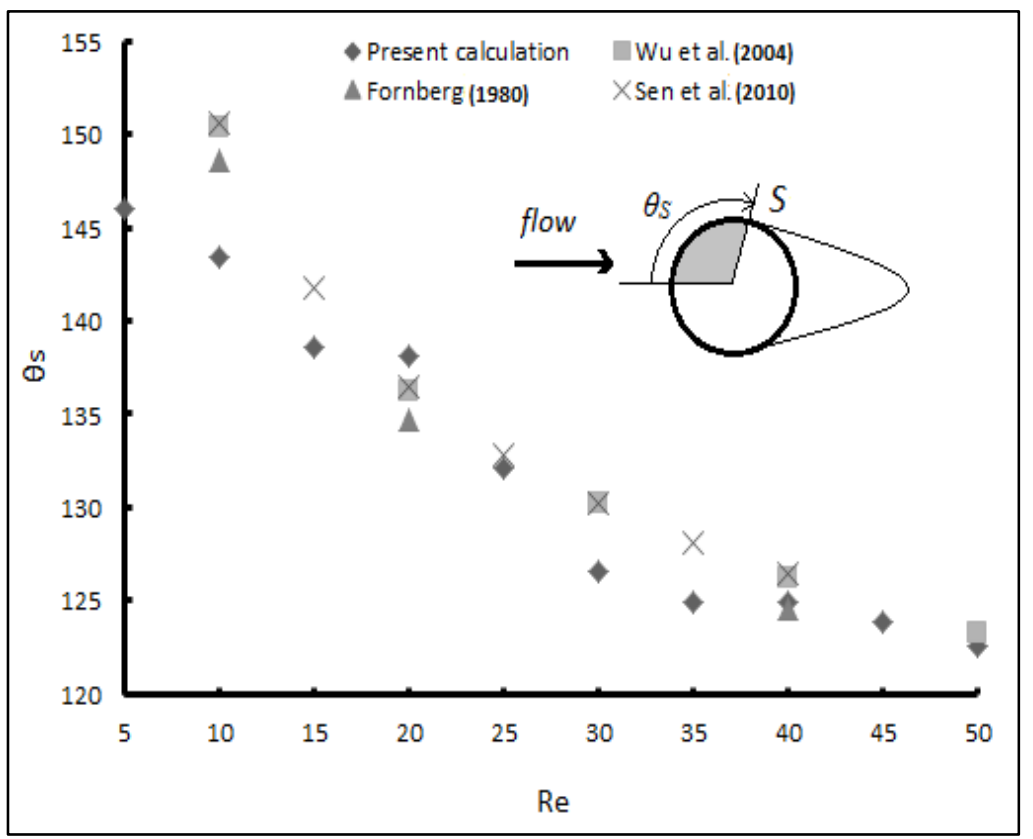

Figure 4. Variation of separation angle with $\operatorname{Re}$ for $B R=0.23077$.

\section{Separation Bubble}

Irrespective of blockage and boundary conditions, the wake of a cylinder for steady flow is closed and symmetric about the free stream flow direction, as is evident in Figure 5. As the 
eddies are symmetric about the horizontal axis, the lift force of the cylinder is expected to be zero. The separation bubble length increases with increasing $R e$ (shown in Figure 6) as the separation point travels upstream with increasing $R e$, which delays the reattachment of the separated flow at the downstream of the cylinder (Sen et al., 2010; Fornberg, 1980). But this rise is up to a certain $R e$ (around 280 from the results of Fornberg (1980)), then it reduces with increasing $R e$. Our present analysis does not cover that region as it is limited up to $R e 50$. The length $L$ is taken from the center of the cylinder and let $d$ be the radius of the cylinder. The proposed linear relationship between the separation bubble length and $R e$ is

$$
L / d=0.0048+0.1655 R e
$$

which is in the range of $5 \leq R e \leq 40$ and for $B R=0.23077$ with root-mean-square error of 0.0085. Fornberg (1980), Park et al. (1998) and Sen et al. (2010) found similar results for steady flow over a cylinder.

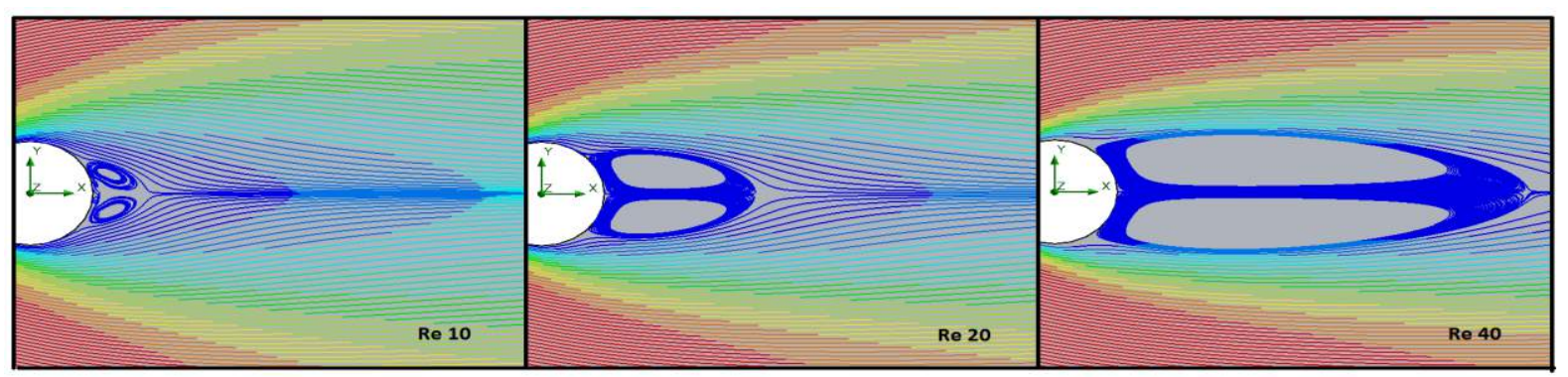

(a)

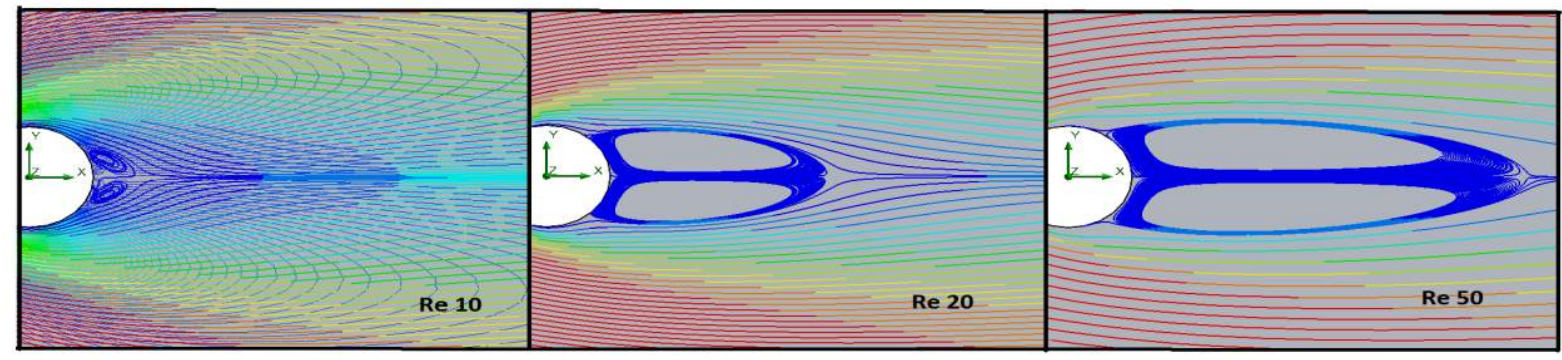

(b)

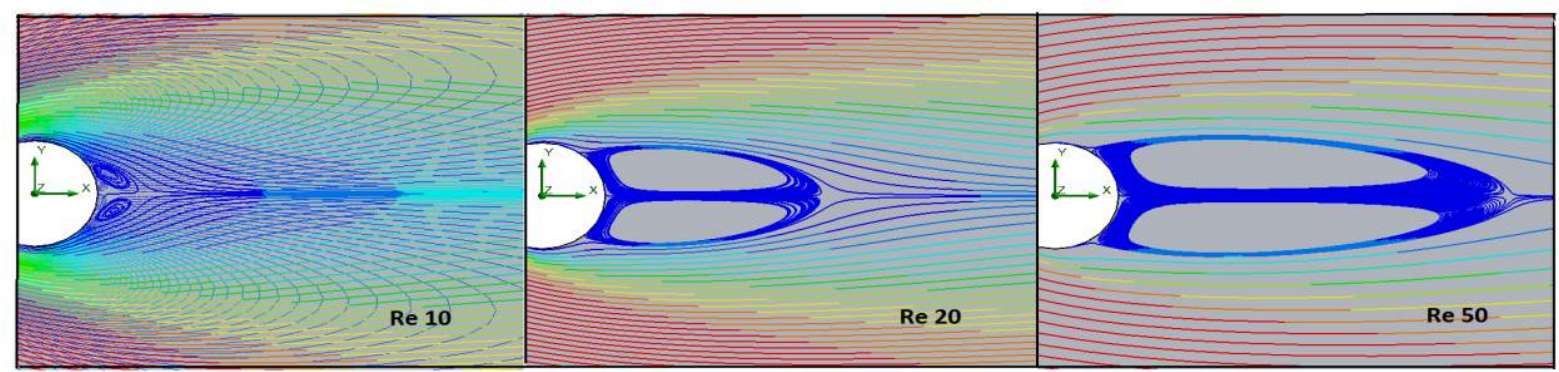

(c)

Figure 5. Symmetric separation bubble at (a) $B R=0.23077$, (b) $B R=1 / 30$ and (c) $B R=1 / 50$. 


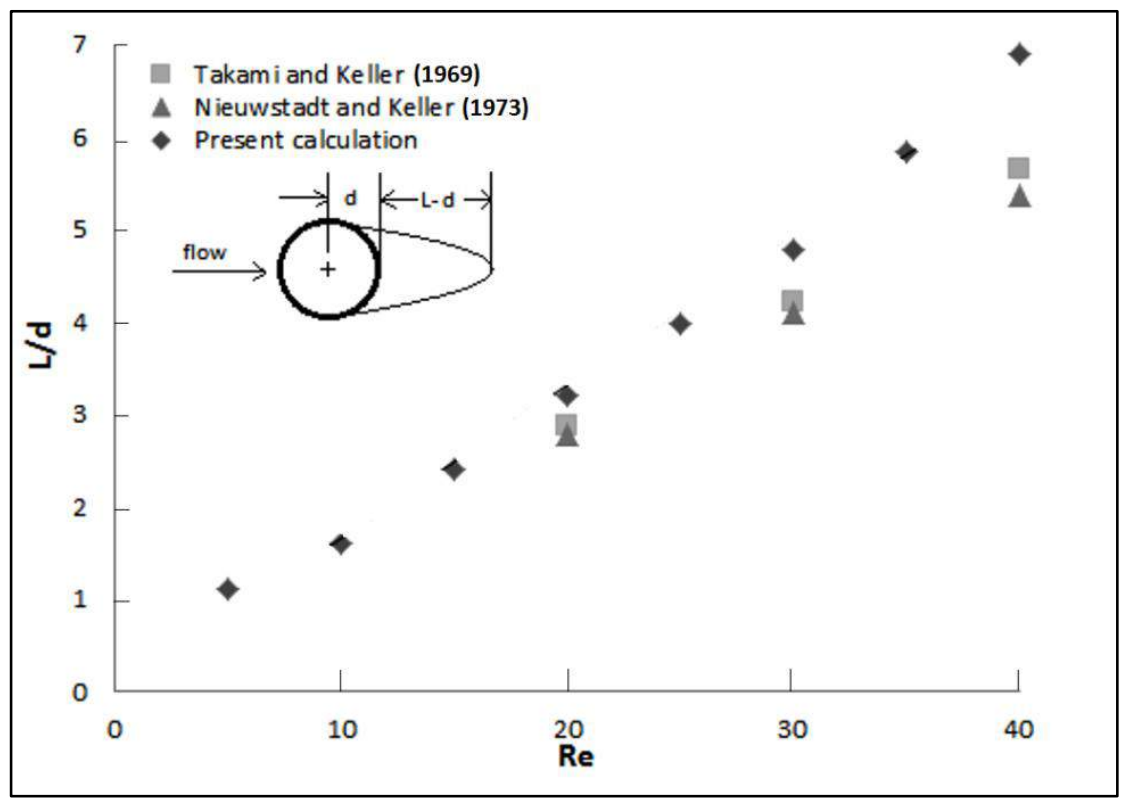

Figure 6. Variation in size of separation bubble at different $R e$ for $B R=0.23077$

\section{Coefficient of Drag at Different Reynolds Number}

The streamlines at different $R e$ and blockage ratios are asymmetric about the vertical axis (or the axis perpendicular to the flow direction), resulting in an unbalanced force distribution between upstream and downstream of the cylinder, which leads to a certain pressure drag towards the flow direction. For a $B R$, the coefficient of drag $C_{D}$ decreases with increasing $R e$ because, with increasing $R e$, the center of the low pressure zone moves downstream, decreasing the suction effect of the low pressure zone on the cylinder towards the flow direction. The low pressure zone is visible as eddies at the downstream. The variation of $C_{D}$ at different $R e$, presented as an empirical relation of $C_{D^{-}} R e$, can be obtained with a root-mean-square error of 0.0066 in the range of $5 \leq R e \leq 50$ and for $B R=0.23077$ as

$$
C_{D}=0.2604+5.3232 R e^{-0.5}
$$

This expression adheres well to the expression provided by Sen et al. (2010) for numerical calculation of steady flow over the cylinder, which is $C_{D}=0.26+7.89 R e^{-0.5}$ in the range of $15 \leq R e \leq 40$ and for $B R=0.01$.

\section{CONCLUSIONS}

The separation phenomena for flow over a circular cylinder are studied numerically for steady flow for Reynolds number up to 50. The onset of separation and subsequent development of the initial wake, the effect of the blockage ratio on the separation angle, the separation bubble length and variation of the separation bubble length with respect to $R e$ and the blockage ratio, variation of the coefficient of drag with $R e$ etc. are extensively studied. These phenomena are expressed numerically by empirical equations as functions of the Reynolds number. It is evident that the point at which the separation occurs moves upstream with increasing $R e$. The blockage ratio has an effect on the flow parameters and the position of the separation point at lower $R e$. With decreasing blockage ratio, the deviation of the separation angle at a definite $R e$ decreases, i.e. the effect of the upper and 
lower wall on the flow over the cylinder decreases. The separation bubble formed at the downstream of the cylinder is symmetric about the horizontal axis irrespective of $R e$ and the blockage ratio. The length of the separation bubble increases with increasing $R e$ and as the center of low pressure zone which is responsible for the separation drag moves further downstream, which results in decrease in the drag coefficient.

\section{REFERENCES}

Fey, U., König, M., \& Eckelmann, H. (1998). A new Strouhal-Reynolds-number relationship for the circular cylinder in the range $47>\operatorname{Re}>2 \times 10^{5}$. Physics of Fluids, $10,1547-1549$.

Fornberg, B. (1980). A numerical study of steady viscous flow past a circular cylinder. Journal of Fluid Mechanics, 98, 819-855.

Hirsch, C. (1988). Numerical computation of internal and external flows. Chichester, England: John Wiley and Sons.

Nieuwstadt, F. \& Keller, H. B. (1973). Viscous flow past circular cylinders. Journal of Fluid Mechanics, 1, 59-71.

Nisi, H. \& Porter, A. W. (1923). On eddies in air. Philosophical Magazine, 46, 754-768.

Park, J., Kwon, K., \& Choi, H. (1998). Numerical solutions of flow past a circular cylinder at Reynolds numbers up to 160. KSME International Journal, 12, 1200-1205.

Roache, P. J. (1998). Technical Reference of Computational Fluid Dynamics. Albuquerque, New Mexico, USA: Hermosa.

Sen, S., Mittal, S., \& Biswas, G. (2010). Numerical simulation of steady flow past circular cylinder. Proceedings of the 37th National \& 4th International Conference on Fluid Mechanics and Fluid Power, IIT Madras, Chennai, India, December 16-18.

Takami, H. \& Keller, H. B. (1969). Steady two-dimensional viscous flow of an incompressible fluid past a circular cylinder. Physics of Fluids, 12(2), 51-56.

Taneda, S. (1956). Experimental investigation of the wakes behind cylinders and plates at low Reynolds numbers. Journal of the Physical Society of Japan, 11, 302-307.

Williamson, C. H. K. \& Brown, G. L. (1998). A series in (1/Re) to represent the StrouhalReynolds number relationship of the cylinder wake. Journal of Fluids and Structures, 12, 1073-1085.

Williamson, C. H. K. (1996). Vortex dynamics in the cylinder wake. Annual Review of Fluid Mechanics, 28, 477-539.

Wu, M. H., Wen, C. Y., Ten, R. H., Weng, M. C., \& Wang, A. B. (2004). Experimental and numerical study of the separation angle for flow around a circular cylinder at low Reynolds number. Journal of Fluid Mechanics, 515, 233-260. 